$11-8-2003$

\title{
Mode Structure of Diffusive Transport in Hydroxypropylcellulose:Water
}

\author{
George D.J. Phillies \\ Worcester Polytechnic Institute, phillies@wpi.edu \\ Robert O. Connell \\ Paul Whitford \\ Kiril A. Streletzky \\ Cleveland State University, K.STRELETZKY@csuohio.edu
}

Follow this and additional works at: https://engagedscholarship.csuohio.edu/sciphysics_facpub

Part of the Physics Commons

How does access to this work benefit you? Let us know!

Publisher's Statement

(c) 2003 American Institute of Physics.

\section{Repository Citation}

Phillies, George D.J.; Connell, Robert O.; Whitford, Paul; and Streletzky, Kiril A., "Mode Structure of Diffusive Transport in Hydroxypropylcellulose:Water" (2003). Physics Faculty Publications. 256.

https://engagedscholarship.csuohio.edu/sciphysics_facpub/256

This Article is brought to you for free and open access by the Physics Department at EngagedScholarship@CSU. It has been accepted for inclusion in Physics Faculty Publications by an authorized administrator of

EngagedScholarship@CSU. For more information, please contact library.es@csuohio.edu. 


\section{Mode structure of diffusive transport in hydroxypropylcellulose:water}

George D. J. Phillies, Robert O'Connell, Paul Whitford, and Kiril A. Streletzky

Citation: 119, 9903 (2003); doi: 10.1063/1.1615968

View online: http://dx.doi.org/10.1063/1.1615968

View Table of Contents: http://aip.scitation.org/toc/jcp/119/18

Published by the American Institute of Physics

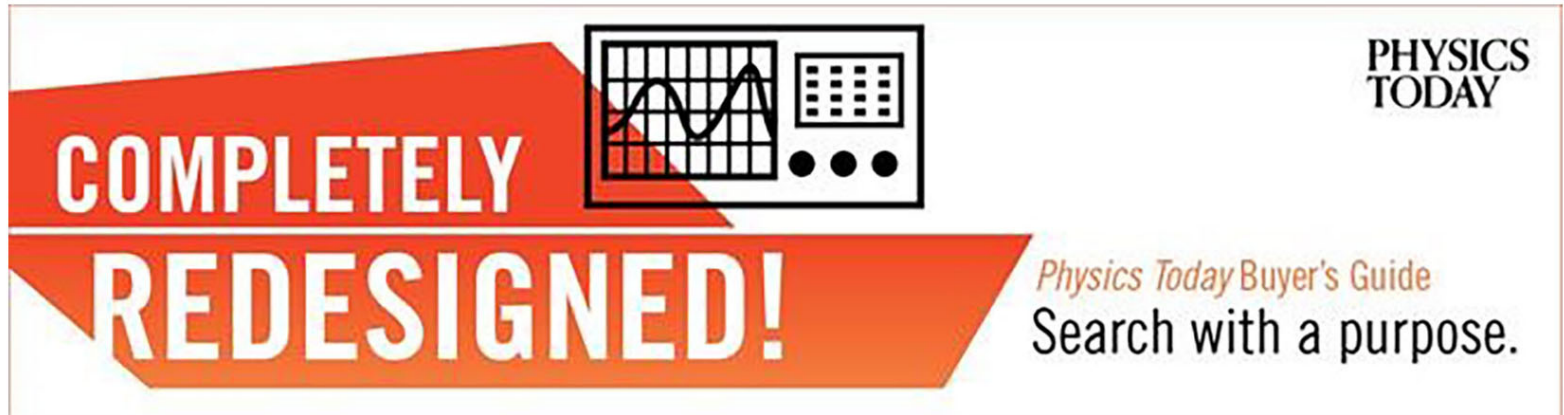




\title{
Mode structure of diffusive transport in hydroxypropylcellulose:water
}

\author{
George D. J. Phillies, ${ }^{\text {a) }}$ Robert O'Connell, Paul Whitford, and Kiril A. Streletzky \\ Department of Physics, Worcester Polytechnic Institute, Worcester, Massachusetts 01609
}

(Received 22 May 2003; accepted 13 August 2003)

\begin{abstract}
A systematic analysis of the mode structure of diffusive relaxations in $1 \mathrm{MDa}$ hydroxypropylcellulose(HPC):water is presented. New methods and data include (1) use of integral spectral moments to characterize nonexponential decays, (2) spectra of small probes in concentrated HPC solutions, (3) temperature dependence of the mode structure, and (4) comparison of optical probe spectra and spectra of probe-free polymer solutions. We find that (1) probe and polymer relaxations are in general not the same; (2) the apparent viscometric crossover near $c_{t} \approx 6 \mathrm{~g} / \mathrm{l}$ is echoed by probe behavior; (3) our HPC solutions have a characteristic dynamic length, namely the $50 \mathrm{~nm}$ length that matches the polymer's hydrodynamic radius; (4) characterization of spectral modes with their mean relaxation time affords simplifications relative to other characterizations; and (5) contrary to some expectations, Stokes-Einsteinian behavior (diffusion rate determined by the macroscopic viscosity) is not observed, even for large probes in relatively concentrated solutions. We propose that the viscometric and light scattering effects found in HPC solutions at elevated concentrations reflect the incipient formation of a generalized Kivelson [S. A. Kivelson et al., J. Chem. Phys. 101, 2391 (1994)] glass. (c) 2003 American Institute of Physics.
\end{abstract}

[DOI: $10.1063 / 1.1615968]$

\section{INTRODUCTION}

Our objective here is to advance understanding of the nature of polymer dynamics in nondilute solutions. Polymer motions in solution are potentially modulated by short- and long-range hydrodynamic interactions between neighboring chains, by topological (chain noncrossing) constraints, and by other longer-range interactions such as van der Waals forces between neighboring chains. Additional issues arise if the polymers are polyelectrolytes. The focus of this paper is experimental. We examine the effect of polymer solutions on the motion of mesoscopic probe particles over a range of temperatures, distances, and time scales, and compare our findings with other dynamic information.

Hydroxypropylcellulose (HPC) is a semiflexible, nonionic, water soluble polymer whose physical properties have been studied extensively. Experimental techniques that have been applied to hydroxypropylcellulose solutions, as described below, include static light scattering, viscometry, optical probe diffusion, fluorescence relaxation after photobleaching (FRAP), and quasielastic light scattering spectroscopy (QELSS). Hydroxypropylcellulose has a persistence length of ca. 100. ${ }^{1,2}$ At elevated concentrations it undergoes a phase transition to a lyotropic phase. For the nominal $1 \mathrm{MDa}$ HPC sample studied here (same supplier and lot number), Mustafa et al. report on the basis of static light scattering a molecular weight $\mathrm{t}^{3}$ of $855 \mathrm{kDa}$ and a radius of gyration $^{4}$ of $124 \pm 34 \mathrm{~nm}$. For an HPC of the same inferred $M_{w}$, Yang and Jamieson ${ }^{5}$ report a radius of gyration of 95 $\mathrm{nm}$ and an average hydrodynamic radius $1 /\left\langle R_{h}^{-1}\right\rangle$ of $49 \mathrm{~nm}$.

Phillies and Quinlan ${ }^{6}$ reported detailed measurements of the low-shear viscosity $\eta$ of HPCs (nominal molecular

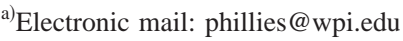

weights 300,1000 , and $1150 \mathrm{kDa}$ ) in aqueous solution at more than 40 concentrations $c$, for $\eta$ up to $3 \times 10^{5} \mathrm{cP}$. Phillies and Quinlan identified in $\eta(c)$ a transition concentration $c_{t}$. For $c<c_{t}$, the viscosity has a stretchedexponential $\left(\exp \left(-\alpha c^{\nu}\right)\right)$ concentration dependence. For $c$ $>c_{t}, \eta$ has a power-law $\left(c^{x}\right)$ concentration dependence. Numerical differentiation to determine $d \log (\eta) / d \log (c)$ finds that the bilogarithmic derivative increases linearly below the transition concentration, is nearly constant above the transition concentration, and is continuous through the transition. The transition between concentration dependences is thus sharp (no crossover region) and analytic (first derivative continuous).

Optical probe diffusion, invented three decades ago by Hallett and Gray ${ }^{7}$ and Turner and Hallett, ${ }^{8}$ is an extension of conventional quasi-elastic light scattering spectroscopy. In this method, mesoscopic, strongly scattering probe particles are mixed with an equilibrium, weakly scattering solution. Light scattering spectroscopy or a related technique is used to measure the diffusive motion of the particles. The solution's dynamic properties are inferred from the probe's dynamics. Turner and Hallett viewed their method as a logical extension of the classical ultracentrifuge studies of Laurent et al. ${ }^{9,10}$ who observed the sedimentation of colloidal particles through solutions of various polymers.

There are extensive optical probe studies of aqueous hydroxypropylcellulose solutions. Brown and Rymden, ${ }^{11}$ using light scattering spectroscopy, found that polystyrene latex in hydroxypropylcellulose:water forms clusters, aggregation being prevented by the addition of 0.15 wt. \% Triton X-100 to the solutions. Russo et al., ${ }^{12}$ using 79 and $181 \mathrm{~nm}$ latex probes, and Mustafa and Russo, ${ }^{3}$ using $91 \mathrm{~nm}$ probes, confirmed that addition of Triton X-100 suppresses the aggregation of polystyrene probes that would otherwise be induced 
by HPC. Russo et al. ${ }^{12}$ demonstrated that the order of mixing of probes and Triton X-100 with the polymer solution is not significant, thereby showing that the process of adsorption to the probes is reversible. They ${ }^{12}$ also found that light scattering spectra of probe:polymer solutions continue to be bimodal after adding Triton $\mathrm{X}-100$. The relaxation rates of the two modes depend on the scattering vector $q$ linearly in $q^{2}$. Yang and Jamieson ${ }^{5}$ used light scattering spectroscopy to study 120,210 , and $350 \mathrm{~nm}$ probes in aqueous solutions of 110 , 450, 620, and $950 \mathrm{kDa}$ HPC, using TX-100 to suppress polymer binding. Yang and Jamieson found that the probe particles diffused more rapidly than expected from $\eta$, especially for the two larger polymers and for the smaller probes. Diffusion of polystyrene latex probes through HPC solutions has since been studied in this laboratory, ${ }^{13-19}$ as discussed below.

Mustafa et al. $^{4}$ used fluorescence recovery after photobleaching (FRAP) to examine the diffusion of fluorescein dye through 103, 292, and $855 \mathrm{kDa}$ HPC. Mustafa et al. found that $D$ falls exponentially in HPC concentration out to concentrations $c>700 \mathrm{~g} / \mathrm{l}$. $D$ of the dye was nearly independent of polymer molecular weight $M$. FRAP infers a single diffusion coefficient, corresponding to motions over very long distances. From $D$ a microscopic viscosity $\eta_{\mu}$ can be inferred from the Stokes-Einstein equation

$$
D=\frac{k_{B} T}{6 \pi \eta_{\mu} r},
$$

where $k_{B}$ is Boltzmann's constant, $T$ is the absolute temperature, and $r$ is the probe radius.

$\mathrm{Bu}$ and Russo ${ }^{20}$ used FRAP to measure the diffusion of dye molecules and dye-labeled dextrans, radii $0.5-55 \mathrm{~nm}$, through $60 \mathrm{kDa}, 300 \mathrm{kDa}$, and $1 \mathrm{MDa}$ HPC solutions. Increasing the HPC molecular weight retards probe motion, the retardation being more marked for large probes than for small probes. The solvent viscosity is in general much less than the macroscopic solution viscosity. With increasing probe size, $\eta_{\mu}$ from FRAP increases from a small multiple of the solvent viscosity toward the macroscopic solution viscosity $\eta$, but $\eta_{\mu}$ is always less than $\eta$. For $55 \mathrm{~nm}$ dextrans-the largest probes studied by $\mathrm{Bu}$ and Russo-in $300 \mathrm{kDa}$ HPC, $\eta_{\mu}$ is a factor of 2 or more smaller than $\eta$.

Recently, Phillies et al. ${ }^{21}$ completed a study of light scattering spectra of probe-free aqueous HPC solutions. Phillies et al. find that light scattering spectra of HPC itself in solution are complex. Below $c_{t}$, spectra are bimodal; both modes relax more rapidly as concentration is increased. At $c_{t}$, QELSS spectra of HPC:water solutions gain a third, extremely long-lived relaxational mode.

The remainder of this paper presents new results on probe diffusion in HPC:water. The next section describes an alternative path to characterizing complex light scattering spectra, namely evaluation of the time moments $M_{i}$ of the spectrum. The zeroth time moment of a spectral mode is shown to give its mean relaxation time. The third section of the paper presents new measurements on optical probe diffusion in HPC solutions using small probes, and on the temperature dependence of probe diffusion. The fourth section of the paper applies the time moment method to reinterpret prior data of Streletzky et al., ${ }^{13-19}$ and unify it with new data reported here on smaller probes at high concentration and on other data on these solutions. Finally, we bring together the various results and consider what the mode structure reveals of polymer dynamics.

\section{METHODS}

We studied quasielastic light scattering spectra of mesoscopic probes in aqueous solutions of nominal 1 MDa hydroxypropylcellulose (HPC) (Scientific Polymer Products). Sample cells were transparent plastic or glass fluorimeter cells, four sides polished, cleaned with dust-free deionized water and dried with dust-free nitrogen prior to introducing the samples into the cells. Samples for light scattering spectroscopy were made with dust-free deionized water, and introduced into the scattering cells via passage through microporous filters having pore diameters $0.2-1.2 \mu \mathrm{m}$. At elevated concentrations the extremely high solution viscosity makes it impossible to pass solutions through small-pore filters. To eliminate possible artifacts arising from changes in filter pore diameter as the concentration is increased, we used one filter size for all solutions in an experimental series, even though the more dilute solutions could have been clarified with a smaller-pore-size filter. Some solutions had added to them optical probes, namely carboxylate-modified polystyrene probes (Seradyn, Interfacial Dynamics) having nominal diameters $20,35,87$, or $189 \mathrm{~nm}$. To prevent the polymer from binding to the probes, all solutions included 0.2 wt. $\%$ Triton X-100.

For temperature control, sample cells were mounted in massive copper blocks through which thermostatted ( $\pm 0.1 \mathrm{C})$ water was circulated. Light scattering spectra were obtained using argon-ion lasers operated at $515 \mathrm{~nm}$, generally at powers of several hundred $\mathrm{mW}$ or less. The laser light was brought to a focus in the scattering volume. Window flare was eliminated and angular resolution was obtained by placing a pair of nearly closed irises between the scattering cell and the photomultiplier tube. Photocount signals were sent via a preamplifier/discriminator/pulse shaper into an ALV-5000 or BI-2030AT digital correlator. To avoid artifacts arising from photomultiplier tube afterpulsing, the first $2 \mu \mathrm{S}$ of the correlation function was not used in spectral fitting. The shortest-lived relaxations observed here lasted more than $100 \mu \mathrm{S}$, so this exclusion has no significant effect on analysis of the measured spectra.

Spectra were fit to selected forms via nonlinear-leastsquares methods using the simplex algorithm. The forms specify the field correlation function $g^{(1)}(t)$, which is related to the measured spectrum $g^{(2)}(t)$ via

$$
g^{(2)}(t)=A\left(g^{(1)}(t)\right)^{2}+B,
$$

where $A$ is the spectral amplitude and $B$ is the baseline to which the spectrum decays at long times.

We are here concerned with numerical characterization of relaxational modes whose forms are well-approximated by stretched exponentials in time. It is not claimed that the stretched-exponential approximation is unique or perfect. For 
applications here, the only requirement is that the approximation be accurate numerically to within noise over a full range of delay times. A single stretched-exponential mode $i$ has as its field correlation function

$$
g_{i}^{(1)}(q, t)=A_{i} \exp \left(-\theta_{i} t^{\beta_{i}}\right)
$$

or equivalently

$$
g_{i}^{(1)}(q, t)=A_{i} \exp \left(-\left(t / \tau_{i}\right)^{\beta_{i}}\right) .
$$

Here $g_{i}^{(1)}(q, t)$ arises from mode $i, A_{i}$ is a mode amplitude, and $\beta_{i}, \theta_{i}$, and $\tau_{i}$ are the mode's stretching exponent, decay pseudorate, and decay pseudotime, respectively. The $\theta_{i}$ are pseudorates, not true rates, because their units are $1 /(\text { time })^{\beta_{i}}$, not $1 /$ time. While it has units time, ${ }^{1} \tau_{i}$ is a pseudotime because it functionally enters $g_{i}^{1}(q, t)$ as the noninteger power $\tau_{i}^{\beta_{i}}$ whose dimensions are (time) $)^{\beta_{i}}$.

The relationship between the $g^{(1)}$ and the timedependent positions $\mathbf{r}_{i}(t)$ of the $N$ scattering particles constrains properties of $\theta_{i}$ and $\tau_{i}$. For dilute scattering particles in a (perhaps complex) nonscattering background fluid,

$$
g^{(1)}(q, t)=A\left\langle\sum_{i=1}^{N} \exp \left(i \mathbf{q} \cdot\left(\mathbf{r}_{i}(t)-\mathbf{r}_{i}(0)\right)\right)\right\rangle .
$$

Here $\mathbf{q}$, with magnitude $q$, is the scattering vector, and the brackets $\langle\cdots\rangle$ denote an ensemble average. For nondilute scattering particles a similar but more complex form is found. ${ }^{22}$ On Taylor expansion in $\mathbf{q}$ of the exponential, noting that terms odd in the displacement vanish by symmetry, replacing each probe's displacement between 0 and $t$ by its velocity $\mathbf{v}_{i}(s)$ as integrated over the time interval, and extracting constants from the time and ensemble averages, one has

$$
\begin{aligned}
g^{(1)}(q, t) & \\
= & 1-\frac{1}{2} q^{2}\left\langle\int_{0}^{t} d s_{1} \int_{0}^{t} d s_{2}\left(\hat{q} \cdot \mathbf{v}_{i}\left(s_{1}\right)\right)\left(\hat{q} \cdot \mathbf{v}_{i}\left(s_{2}\right)\right)\right\rangle \\
& +\frac{1}{4 !} q^{4}\left\langle\int_{0}^{t} d s_{1} \int_{0}^{t} d s_{2} \int_{0}^{t} d s_{3} \int_{0}^{t} d s_{4}\right. \\
& \left.\times\left(\hat{q} \cdot \mathbf{v}_{i}\left(s_{1}\right)\right)\left(\hat{q} \cdot \mathbf{v}_{i}\left(s_{2}\right)\right)\left(\hat{q} \cdot \mathbf{v}_{i}\left(s_{3}\right)\right)\left(\hat{q} \cdot \mathbf{v}_{i}\left(s_{4}\right)\right)\right\rangle-\cdots
\end{aligned}
$$

with $\hat{q}=\mathbf{q} / q$ being the unit vector, and the $s_{i}$ being time variables. The field correlation function $g^{(1)}(q, t)$ is thus a power series in $q^{2}$, the coefficients of $q^{2}$ being terms that may depend on $t$ but are independent of $q$ and (by symmetry) are independent of the direction of $\hat{q}$.

The corresponding expansion of Eq. (3) as a power series in $\theta$ does not necessarily correspond term-by-term with the series in $q^{2}$, but every term in Eq. (6) beyond the leading " 1 " is a power of $q^{2}$, so if the two series are convergent the lead dependence of $\theta$ on $q$ must also be as $q^{2}$ or a positive integer power thereof. The lead dependence of $\tau$ on $q$ follows from the relation $\tau=\theta^{-\beta}$, namely $\tau$ must be proportional to the nonintegral power $q^{-2 / \beta}$. The above analysis of $\theta$ and $\tau$ cannot in general be inverted by expanding Eq. (4) as a power series in $\tau^{-1}$-which would imply $\tau \sim q^{-2}$-because $\beta$ is not an integer, so Eq. (4) is not analytic in, and does not have a Taylor expansion in, $\tau$.

In our systems, as seen below, the individual spectral modes are indeed fit accurately by stretched-exponential forms. However, from the above $\theta_{i}$ and $\tau_{i}$ have the curious features that the first has dimensions time raised to a noninteger power that varies from spectrum to spectrum, while the latter depends on scattering vector $q$ to a noninteger power that varies from spectrum to spectrum. These peculiar features are unattractive for systematic analyses.

To avoid these features, we here introduce the characterization of quasielastic light scattering spectra via spectral time moments. Spectral time moments are a natural extension of the exponential moment integral

$$
M_{n} \equiv \int_{0}^{\infty} d t t^{n} \exp (-\Gamma t)=\frac{\gamma(1+n)}{\Gamma^{1+n}} .
$$

Here $\Gamma$ is a true decay rate of a pure exponential, $n$ is the order of the moment $M_{n}$, and $\gamma(1+n)=n !$ is the gamma function, nontraditionally notated to distinguish it from the spectral decay rate $\Gamma$. For a single-exponential decay one has $\Gamma=1 / M_{0}$ and $\Gamma^{2}=1 / M_{1}$.

An arbitrary monotonically decaying spectrum can be written as a sum of exponentials, viz.

$$
g^{(1)}(t)=\int_{0}^{\infty} d \Gamma A(\Gamma) \exp (-\Gamma t),
$$

where $A(\Gamma)$ is the fractional decay amplitude for decay rate $\Gamma$. It is convenient to normalize $A$ as $\int_{0}^{\infty} A(\Gamma) d \Gamma=1$.

From the above, the $n$th integral time moment of $g(t)$ is

$$
M_{n} \equiv \int_{0}^{\infty} d t t^{n} g^{(1)}(t)=\int_{0}^{\infty} d \Gamma A(\Gamma) \frac{\gamma(1+n)}{\Gamma^{1+n}},
$$

so that $M_{n} / n ! \equiv\left\langle\Gamma^{-n-1}\right\rangle$. If $A(\Gamma)$ is normalized, $M_{0}$ $=\left\langle\Gamma^{-1}\right\rangle$ is the mean relaxation time.

Spectral time moments are seen to be analogous to the spectral central moments of $A$, which for $n \geqslant 2$ are

$$
K_{n}=\int_{0}^{\infty} d \Gamma A(\Gamma)(\Gamma-\bar{\Gamma})^{n} .
$$

The first few spectral moments are the first few cumulants of $A$, while the time moments are the negative $\left(\Gamma^{n}, n<0\right)$ moments of $A$. For the negative moments to be convergent, $A(\Gamma)$ must be sufficiently small at small $\Gamma$. The first cumulant is precisely the initial slope $\lim _{t \rightarrow 0} d \log \left(g^{(1)}\right)(t) / d t$ ) of the field correlation function, while higher cumulants correspond to the higher logarithmic derivatives of $g^{(1)}(t)$ in the limit of short time. Contrariwise, the moments correspond to integral averages over $g^{(1)}(t)$. Accurate determination of cumulants requires adequate measurements of $g^{(1)}(t)$ at short times; correspondingly, accurate determination of moments requires adequate measurements of $g^{(1)}(t)$ at long times.

To analyze our spectra with time moments, we characterize our spectra as the sum of a small number of spectral modes, each of which is described accurately by a fewparameter algebraic form. We fit spectra to these forms via nonlinear least squares and then integrate the few-parameter 


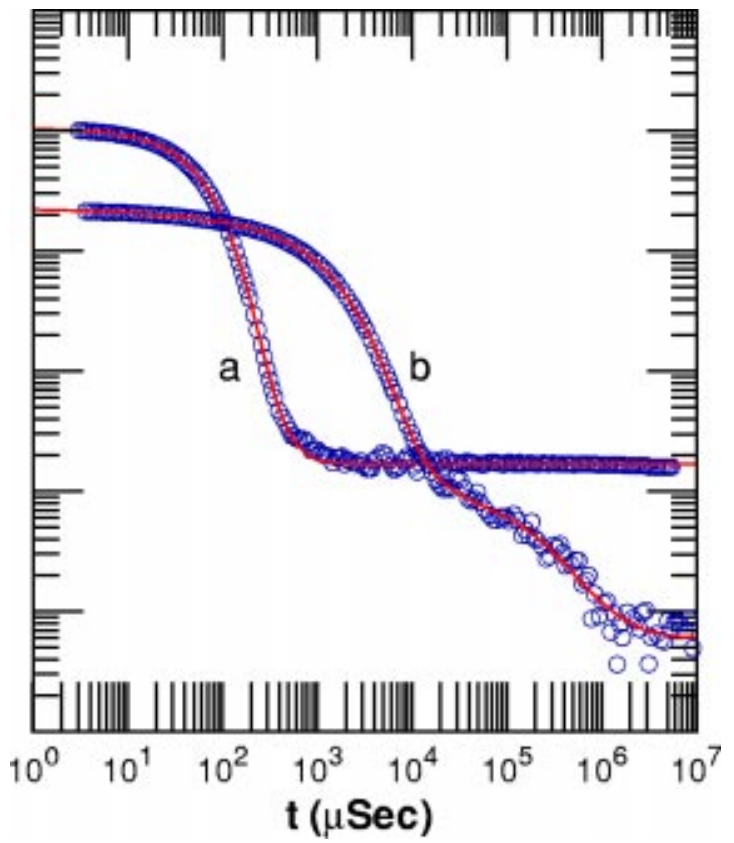

FIG. 1. Spectra of $35 \mathrm{~nm}$ polystyrene probes in (a) 1, (b) $15 \mathrm{~g} / \mathrm{l} \mathrm{HPC}$ solutions, showing the adequacy of a two-stretched-exponential fit to a spectrum of a $1 \mathrm{~g} / \mathrm{l}$ solution, and a successful three-stretched-exponential fit for probes in a $15 \mathrm{~g} / \mathrm{l}$ solution. Spectra given vertical shifts for clarity.

forms analytically, using the least-squares-fitting parameters to compute the mean relaxation times. This approach suppresses some noise effects in the measured $g^{(1)}(t)$.

For the spectra considered here, the fits are to sums of stretched exponentials as seen in Eqs. (3) or (4). Analytic integration of a single stretched-exponential mode shows

$$
M_{0} \equiv \int_{0}^{\infty} d t \exp \left(-\theta t^{\beta}\right)=\gamma(1+1 / \beta) / \theta^{1 / \beta}
$$

for the mean relaxation time of a single stretchedexponential mode. This equation was applied both to new spectra measured for this paper and older spectra from Refs. 13-19.

\section{SMALL PROBES AT ELEVATED CONCENTRATIONS}

Spectra were obtained of $35 \mathrm{~nm}$ diameter probes diffusing in HPC solutions for concentrations $c \in(1,15) \mathrm{g} / \mathrm{l}$. Representative spectra appear in Fig. 1. Below 6 g/l HPC, spectra are fit well by a field correlation function that is a sum of two stretched exponentials, namely a "fast" mode and an "intermediate" mode. Above $6 \mathrm{~g} / \mathrm{l}$ HPC, a third slow stretched-exponential mode appears in the spectrum. Here "fast," "intermediate," and "slow" refer to the mean lifetimes determined from the first time moments of the modes, and not from the initial decay rates of the modes. The initial rates are determined in part by $\beta$ for the modes.

Figure 2 shows the concentration dependences of the first time moment of the modes. At HPC concentrations below $7 \mathrm{~g} / \mathrm{l}$, the fast mode (smaller $M_{0}$ ) dominates the spectrum; it has $\geqslant 80 \%$ of the amplitude. At 1 and $3 \mathrm{~g} / \mathrm{l}$, the fast and intermediate modes are nearly pure exponentials $(\beta$ $\geqslant 0.86$ ); in $5 \mathrm{~g} / \mathrm{l} \mathrm{HPC}$, the intermediate mode broadens considerably.

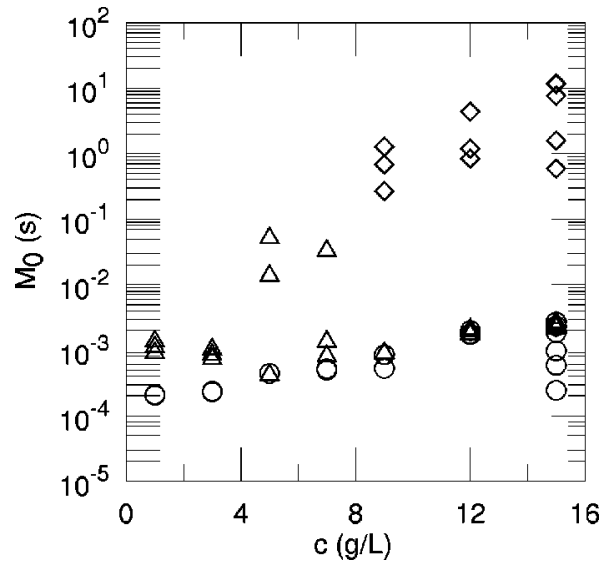

FIG. 2. Spectral moments $M_{0}$ for the modes of $35 \mathrm{~nm}$ polystyrene probes as functions of HPC concentration. A two-stretched-exponential fit (open points) is adequate at lower concentrations ( $c \leqslant 6 \mathrm{~g} / \mathrm{l} \mathrm{HPC})$, but above $6 \mathrm{~g} / \mathrm{l}$ a third, slow mode and a three-stretched-exponential fit (filled points) is required.

At $7 \mathrm{~g} / \mathrm{l}$ and larger, the two faster modes coalesce to approximately the same $M_{0}$, but have very different widths. One mode has $\beta \in(0.85-1.0)$, while the other has $\beta$ $\in 0.5-0.8$. Light scattering does not reveal whether the underlying physical modes have coalesced into a single physical mode whose relaxation is described well as a sum of two stretched exponentials, or whether the two stretched exponentials describe two different, but approximately equally long-lived, relaxation processes. At the same concentrations, a third, very slow and broad mode appears. The new mode has an $M_{0}$ as long as several seconds and an extremely large width, with $\beta$ as small as $0.1-0.2$.

Over the full observed concentration range, $M_{0}$ of the fastest mode increases roughly tenfold; over the same range $M_{0}$ of the intermediate mode perhaps increases perhaps twofold, though with a dip at intermediate concentrations. Determinations of $M_{0}$ of the slow mode are inaccurate, because the relaxation times are approaching instrumental limits, but at concentrations above the initial onset $M_{0}$ of the slow mode appears to be several seconds.

We have examined the temperature dependence of $g^{(1)}(t)$ for $35 \mathrm{~nm}$ probes in HPC solution over the temperature range $6-40^{\circ} \mathrm{C}$. At lower temperatures, water is a neargood solvent for $\mathrm{HPC}$; as $40^{\circ} \mathrm{C}$ is approached $\mathrm{HPC}$ undergoes a pseudotheta transition. Concentrations examined were $2,4,6,8,10,12$, and $15 \mathrm{~g} / \mathrm{l}$. Based on the above observation that spectra reveal two dominant relaxation times at each concentration, these spectra were all fit to sums of two stretched exponentials.

Figure 3 shows representative data on the temperature dependence of $M_{0}^{-1}$ of the fast and intermediate modes for probes in dilute, nondilute, and concentrated HPC solutions. At each concentration, $M_{0}^{-1}$ is linear in $T / \eta_{0}, T$ being the absolute temperature and $\eta_{0}$ being the viscosity of water at temperature $T$. Above $30^{\circ} \mathrm{C}$, in some solutions there were countervailing changes in $\theta$ and $\beta$ as the pseudotheta transition was approached. A modest nonzero intercept is sometimes observed for $\lim _{T / \eta_{0} \rightarrow 0} M_{0}^{-1}$, but the sign of the intercept is not always the same, implying that the apparent 


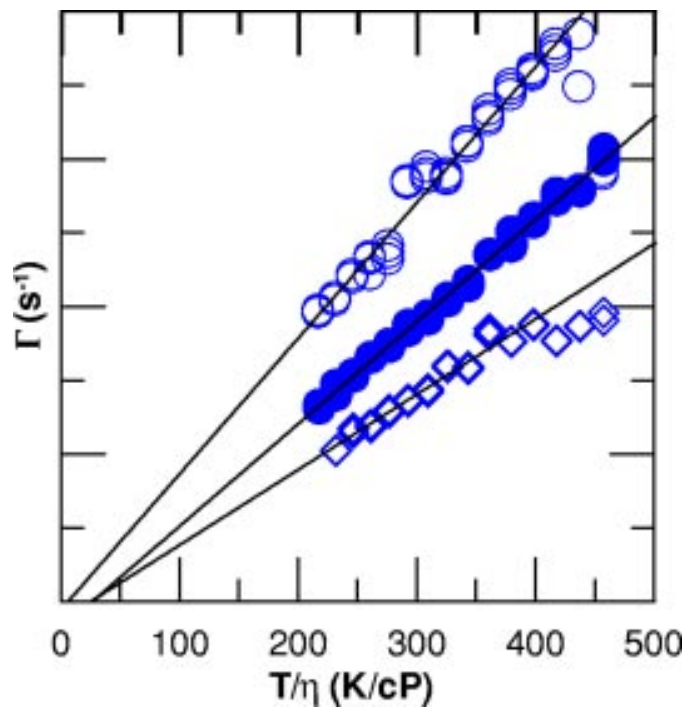

FIG. 3. Temperature dependence of the inverse time moment $\bar{\Gamma} \equiv M_{0}^{-1}$ for spectra of $35 \mathrm{~nm}$ polystyrene probes, showing (a) $2 \mathrm{~g} / \mathrm{l} \mathrm{HPC}$, intermediate mode (open circles), (b) $8 \mathrm{~g} / \mathrm{l} \mathrm{HPC}$, fast mode (circles), and $12 \mathrm{~g} / \mathrm{l} \mathrm{HPC}$, intermediate mode (diamonds). Vertical was rescaled for clarity.

intercepts are simply a consequence of random noise. Our measurements show that mode relaxation is controlled by solvent hydrodynamics, not by some internal nonhydrodynamic process inhering in the polymer.

We have also obtained spectra of probe-free solutions of hydroxypropylcellulose in water. As reported separately, ${ }^{21}$ these solutions give bimodal spectra for $c<6 \mathrm{~g} / \mathrm{l}$. At concentrations above $6 \mathrm{~g} / \mathrm{l} \mathrm{HPC}$, spectra become trimodal, with an additional very slow mode $\left(M_{0}\right.$ on the order of $\left.1 \mathrm{~s}\right)$ becoming visible.

Figure 4 shows the mean relaxation times $M_{0}$ for the scattering modes of HPC solutions. The mean relaxation time of the fast mode decreases moderately with increasing concentration, from $\sim 500 \mu \mathrm{s}$ at low concentration to a plateau near $50 \mu \mathrm{s}$ at concentrations above $6 \mathrm{~g} / \mathrm{l}$. The intermediate mode rate wanders a bit with changing concentration but over the full range studied never moves far from 1-3 ms. Finally, the slow mode appears at concentrations above $6 \mathrm{~g} / \mathrm{l}$,

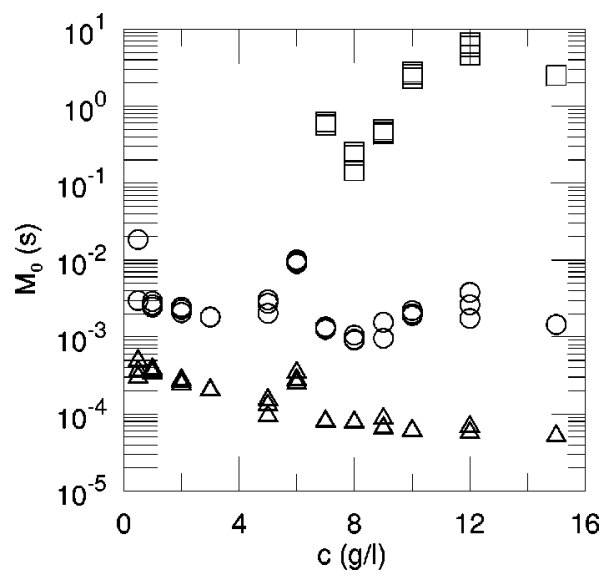

FIG. 4. Mean relaxation times $M_{0}$ for the fast (triangles), intermediate (circles), and slow (squares) optical scattering modes of hydroxypropylcellulose:water, as functions of concentration. increasing with increasing $c$ through the range $0.3-6 \mathrm{~s}$. The fast and intermediate relaxation times measured at $6 \mathrm{~g} / \mathrm{l}$ are markedly larger than those measured at 5 or $7 \mathrm{~g} / \mathrm{l}$. While attempted fits of $6 \mathrm{~g} / \mathrm{l}$ solution spectra to a trimodal spectrum did not resolve a third, slow mode, an incipient slow mode that was still too weak near $5-6 \mathrm{~g} / \mathrm{L}$ to be separately resolved might increase the apparent mean relaxation times of the two visible modes, as seen in the figure.

We also determine the static light scattering intensity $I$ at the scattering angle used to generate Fig. 4. The normalized intensity $I / c$ declines slowly with increasing $c$. There is no signature in the scattering intensity that might reflect the appearance of the slow mode. The slow mode's appearance corresponds to a transfer of intensity from the intensities of the two pre-existing modes, not to the appearance of new intensity in the scattering signal.

Control experiments in which probe and polymer solution spectra were measured with identical laser powers, iris settings, etc., indicate that the polymer spectra are substantial weaker than the probe spectra at all relaxation times, so coincidences between the mean relaxation times of polymer and probe modes do not appear to be artifacts arising from polymer scattering contaminating the probe scattering.

\section{SPECTRAL MOMENTS FOR SMALL AND LARGE OPTICAL PROBES}

Streletzky et al. ${ }^{13-18}$ report light scattering spectra of polystyrene latex probes, diameters $14-455 \mathrm{~nm}$, diffusing through $1 \mathrm{MDa}$ HPC. Large $(R>50 \mathrm{~nm})$ probes were studied for $c$ up to $15 \mathrm{~g} / 1$; small $(R<50 \mathrm{~nm})$ probes were studied only up to $c_{t}$ or so $(7 \mathrm{~g} / \mathrm{l})$. In these references, spectra were parametrized using Eqs. (2)-(4); tables of parameters appear in Ref. 15. We have converted these parameters to the corresponding spectral time moments and reanalyzed the behavior of the spectra.

For small probes (diameter less than $50 \mathrm{~nm}$ ) moment analysis reveals a short-lived, sharp mode $[\beta \in(0.5,1)]$ with mean lifetime $100 \mu \mathrm{s}-1 \mathrm{~ms}$, and a broad mode $[\beta$ $\in(0.2,0.6)]$ with longer lifetime $1-100 \mathrm{~ms}$. The earlier analysis of Streletzky et al. named the modes by the rapidities of their initial decays, a mode with a smaller $\beta$ being "faster" in this sense. For small probes, the "fast" mode of Streletzky et al. is the longer-lived "broad" mode, while the "slow" mode of Streletzky et al. is the shorter-lived (smaller $M_{0}$ even though larger $\beta$ ) "sharp" mode. (For large probes, the "slow" mode of Streletzky et al. is indeed the longer lived; it is also the sharper mode with $\beta=1$.) If Streletzky et al. had named modes based on their $M_{0}$ values rather than their initial decay rates, for small probes the Streletzky et al. "fast" mode would have been termed the "slow" mode and vice versa. We therefore discontinue the use of the Streletzky et al. nomenclature.

For small probes, mode amplitudes depend markedly on $q$. At a $90^{\circ}$ scattering angle, the sharp mode dominates the spectrum, the zero-time fractional amplitude $A_{s}$ of the sharp mode being $0.9-0.95$ at $1 \mathrm{~g} / \mathrm{l} \mathrm{HPC}$, falling to $\sim 0.7$ at the highest HPC concentration ( $7 \mathrm{~g} / \mathrm{l})$ studied by Streletzky et al., with small probes. However, as $q$ is reduced, the 

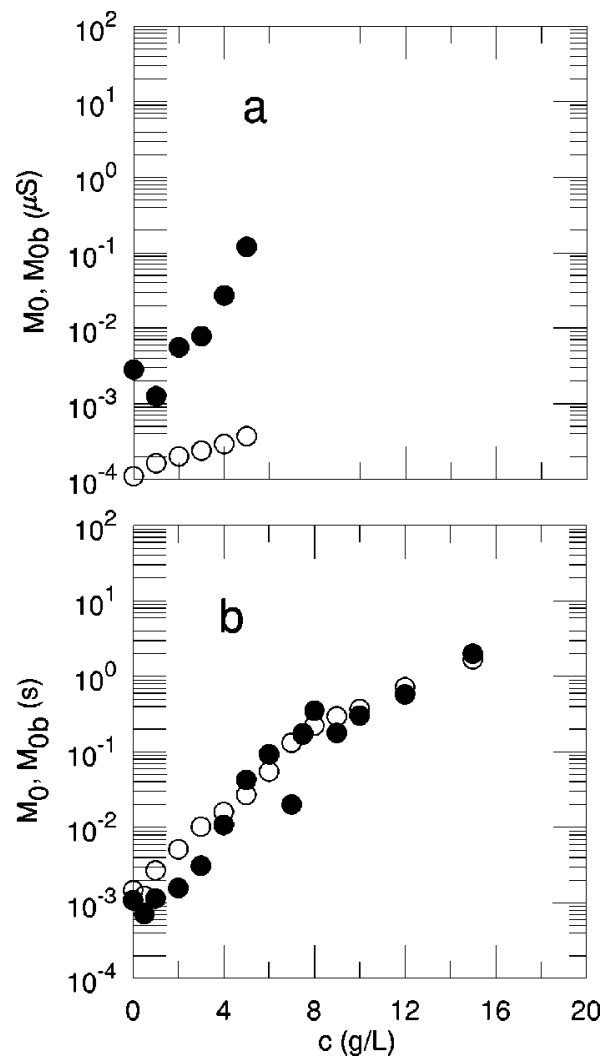

FIG. 5. Mean lifetimes $M_{0}$ and $M_{0 b}$ for the sharp ( $\left.\bigcirc\right)$ and broad (๑) modes of (a) 21 and (b) $189 \mathrm{~nm}$ polystyrene probes as functions of polymer concentration.

longer-lived broad mode becomes dominant, so that $A_{s} \rightarrow 0$ at low $q$.

As seen in Fig. 5(a), for small probes $M_{0}$ of the longerlived broad mode depends on polymer concentration more strongly than does $M_{0}$ of the shorter-lived sharp mode. For large (diameter above $60 \mathrm{~nm}$ ) probes, previous work ${ }^{13-18}$ identified a sharp $(\beta=1)$ "slow" mode and a broad ( $\beta$ falling from 1 to 0.6 or so with increasing $c$ ) "fast" mode. As seen in Fig. 5(b), for large probes in solutions having concentrations below $4 \mathrm{~g} / \mathrm{l}$ the sharp mode has a longer mean lifetime than the broad mode. As was also the case for the 35 $\mathrm{nm}$ spheres, at concentrations above $4 \mathrm{~g} / \mathrm{l}$ HPC the fractional amplitudes and lifetimes of the sharp and broad modes become nearly equal. It is not apparent whether the two modes physically coalesce. Spectra are not fit well by a single stretched exponential. The mean relaxation times of the two modes are about the same, but their line shapes ( $\beta$ values) are very different.

Note that in less concentrated solutions $(c<4 \mathrm{~g} / \mathrm{L})$ the relative mean lifetimes of the sharp and broad modes invert between small and large probes. At lower concentrations: For small probes, the sharp mode is shorter lived than the broad mode, while, for large probes, the sharp mode is longer lived than the broad mode. For all probes, the broad mode depends more strongly on concentration than the sharp mode does.

The dependence of $M_{0}$ on probe diameter $R$ is clarified by Fig. 6, which shows $M_{0}$ of the two modes as functions of $R$ at concentrations 2,4 , and $15 \mathrm{~g} / 1 . M_{0 s}$ of the sharp mode increases with increasing probe diameter. As noted above,

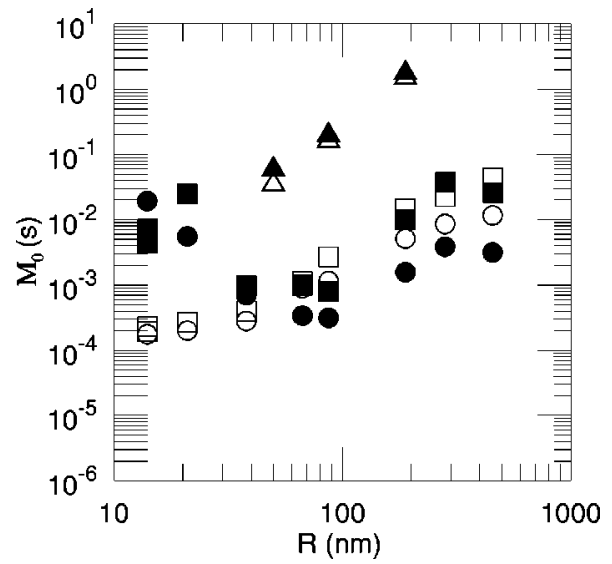

FIG. 6. Mean lifetimes $M_{0}$ for the sharp (open points) and broad (filled points) modes for all probes at concentrations 2 (circles), 4 (squares), and (c) 15 (triangles) g/l, based on data in Ref. 15. All concentrations were not studied with all probes.

systematic size dependences in $15 \mathrm{~g} / \mathrm{l}$ solutions were only obtained for the large probes, for which $M_{0 s} \approx M_{0 b}$.

At lower concentrations, of which the 2 and $4 \mathrm{~g} / \mathrm{l}$ solutions described here are examples: With increasing probe diameter, $M_{0 b}$ of the broad mode at first decreases until it is smaller than $M_{0 s} ; M_{0 b}$ then increases with increasing $R$. In consequence, for each concentration there is a smallest probe diameter $R_{c}$ at which $M_{0 b} \approx M_{0 s}$. While $R_{c}$ might in principle depend on $c$, we find $R_{c}$ is consistently $50-60 \mathrm{~nm}$.

For probes smaller than $R_{c}, M_{0 s}$ increases approximately linearly with increasing $R$. When $R$ is increased above $R_{c}$, with increasing $R$ a transition region of rapidly increasing $M_{0 s}$ is seen. For large probes $(R>180 \mathrm{~nm}), M_{0 s}$ is again approximately linearly proportional to $R$, but the proportionality constant is approximately tenfold larger than it was for small probes.

The mean relaxation times also depend on the experimental scattering vector $q$. For quite large $(189 \mathrm{~nm})$ probes observed $^{15}$ at HPC concentrations 1-3 g/l, the sharp mode was dominant. The inverse mean lifetime $\bar{\Gamma}=M_{0}^{-1}$ of the sharp mode is uniformly linear in $q^{2}$, with $\bar{\Gamma} \rightarrow 0$ as $q^{2} \rightarrow 0$. The weaker (short-lived) mode has an inverse mean lifetime $\bar{\Gamma}_{b}$ that is approximately linear in $q^{2}$, though with significantly more scatter in the data points.

For smaller $87 \mathrm{~nm}$ probes observed ${ }^{15}$ at HPC concentrations of $1,2,4,5$, and $12 \mathrm{~g} / \mathrm{l}, \bar{\Gamma}$ of the sharp $(\beta=1)$ mode depends linearly on $q^{2}$ and goes toward zero at low $q . \bar{\Gamma}_{b}$ of the broad $(\beta \approx 1 / 2)$ mode was again difficult to determine with good precision at low $(c<3 \mathrm{~g} / \mathrm{l}$ HPC) concentrations. At higher concentrations, as seen in Fig. $7(\mathrm{a}), \bar{\Gamma}_{b}$ increases linearly in $q^{2}$.

Streletzky ${ }^{15}$ also observed $q^{2}$ dependences of lightscattering spectra of $20 \mathrm{~nm}$ probes in 1-5 g/l HPC; representative data are in Fig. 7(b). At large $q$, the (shorter-lived) sharp mode is dominant; as $q \rightarrow 0$, most intensity switches to the broad mode. At each concentration studied, the $q^{2}$ dependence of the amplitude changes slope markedly for $q^{2} \approx 2$ $\times 10^{10} \mathrm{~cm}^{-2}$, corresponding to distances $q^{-1} \sim 70 \mathrm{~nm}$. $\bar{\Gamma}$ of the sharp mode scales linearly in $q^{2}$. At lower $q, \bar{\Gamma}_{b}$ of the 

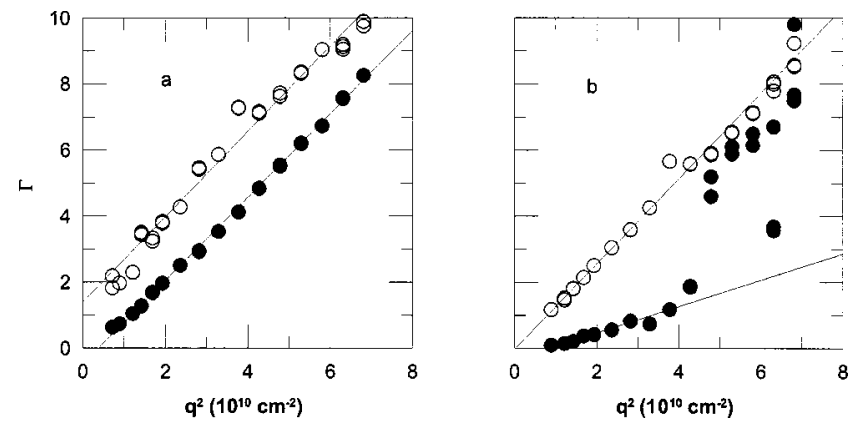

FIG. 7. Angular dependence of the inverse mean relaxation times $\bar{\Gamma}$ of the sharp (O) and broad (O) modes of (a) $87 \mathrm{~nm}$ probes in $5 \mathrm{~g} / \mathrm{l} \mathrm{HPC}$, and (b) $20 \mathrm{~nm}$ probes in $4 \mathrm{~g} / \mathrm{l} \mathrm{HPC}$. (Vertical scales shifted for readability.)

broad mode also increases approximately linearly in $q^{2}$. Over the range $q^{2} \approx 4-5 \times 10^{10} \mathrm{~cm}^{-2}$, corresponding to distances $q^{-1} \approx 50 \mathrm{~nm}, \bar{\Gamma}_{b}$ suddenly increases rapidly with increasing $q^{2}$.

Figure 8 shows the correlation between the mean relaxation times and the solution viscosity. ${ }^{6}$ Following the Stokes-Einsteinian expectation $D \eta \sim$ constant for particles in a simple fluid, we plot $\bar{\Gamma} \eta$ against polymer concentration. For clarity, the figures only show $\bar{\Gamma} \eta$ for six probe diameters, viz., the four diameters $(35,50,87$, and $189 \mathrm{~nm})$ taken to the highest concentration studied, and the near-extremal 14 and $282 \mathrm{~nm}$ diameters. The most concentrated HPC solution, 15 $\mathrm{g} / \mathrm{l}$, has a viscosity $7.3 \times 10^{3} \mathrm{cP}$, so solutions with an 8000 fold variation in viscosity are represented in the figure.

Probe spectra are generally characterized by two modes and relaxation times. In comparing spectra from probes of different size, one must choose which modes of one probe are to be compared with which modes of the other probe. In our earlier papers, the sharp modes were compared as a group, and the broad modes were separately compared as a group. Here we instead compare the shorter-lived modes as a group, and the longer-lived modes as a second group. The shorter-lived mode is the sharp mode of the smaller (14, 35 $\mathrm{nm})$ probes and the broad mode of the larger $(50-282 \mathrm{~nm})$
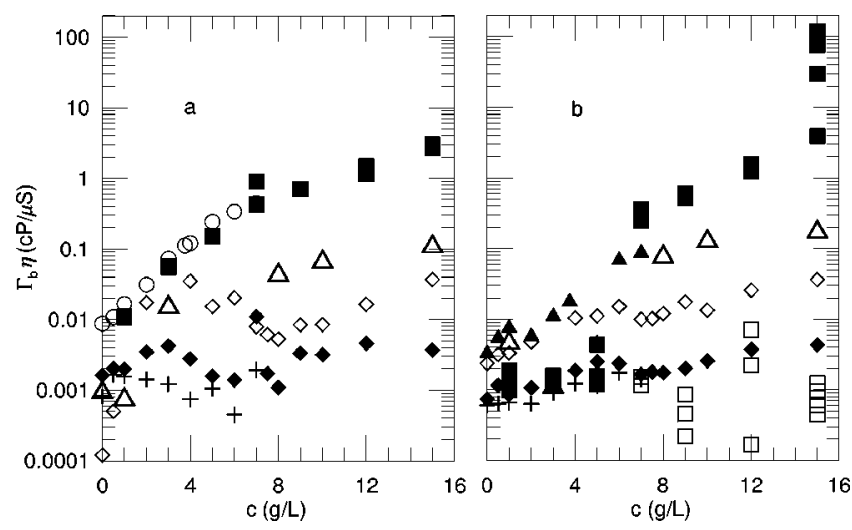

FIG. $8 . \bar{\Gamma} \eta$ for the (a) short-lived and (b) long-lived modes of light scattering spectra of polystyrene probes, diameters 14 (circles), 35 (squares), 50 (triangles), 67 (filled triangles), 87 (open diamonds), 189 (filled diamonds), and 282 (pluses) nm, in HPC:water, as functions of HPC concentration. Open squares are the very slow mode of the $35 \mathrm{~nm}$ probes, whose lifetime is pushing the experimental limits of the apparatus. probes; the longer-lived mode is the broad mode of the smaller probes and the sharp mode of the larger probes. The $35 \mathrm{~nm}$ probes at polymer concentrations above $6 \mathrm{~g} / \mathrm{l}$ have a third, longest-lived, mode.

Figure 8(a) shows $\bar{\Gamma} \eta$ for the shorter-lived mode. $\bar{\Gamma} \eta$ of the 14 and $35 \mathrm{~nm}$ probes have very nearly the same concentration dependences, the $35 \mathrm{~nm}$ probes diffusing modestly more slowly. Over the full concentration range, $\bar{\Gamma} \eta$ of the 35 $\mathrm{nm}$ probes increases nearly 200 -fold. With increasing probe size, the increase in $\bar{\Gamma} \eta$ over the observed concentration range becomes less, e.g., 100-fold for the $50 \mathrm{~nm}$ probes, and only two-fold for the $189 \mathrm{~nm}$ probes, but even for very large probes $\bar{\Gamma} \eta$ is not independent of polymer concentration.

Figure 8 (b) shows $\bar{\Gamma} \eta$ for the longer-lived modes. For the 50, 89, 187, and $282 \mathrm{~nm}$ probes, $\overline{\bar{\Gamma}} \eta$ increases with increasing $c$, by 25 -fold for the $50 \mathrm{~nm}$ probes, nearly 20 -fold for the $89 \mathrm{~nm}$ probes, by more than fourfold for the $189 \mathrm{~nm}$ probes, and by more than twofold for the $282 \mathrm{~nm}$ probes. Above $6 \mathrm{~g} / \mathrm{l}$, the slope $d \log (\bar{\Gamma} \eta) / d c$ appears to decrease. For the $35 \mathrm{~nm}$ probes, $\bar{\Gamma} \eta$ of the third, very slow mode appears to be independent (within experimental scatter, which is large) of polymer concentration.

It might be proposed that Stokes-Einsteinian behavior ( $\bar{\Gamma} \eta$ independent of polymer concentration) should be found for sufficiently large probes at a sufficiently large polymer concentration. We did not find an elevated polymer concentration above which $\bar{\Gamma} \eta$ becomes constant for large probes. In the most concentrated solution studied, in which $\eta$ is more than 8000 times greater than the solvent viscosity, the 50 and $87 \mathrm{~nm}$ probes diffuse more rapidly than expected from $\eta$ and their diameters. Stokes-Einsteinian behavior is more closely approached when the probe diameter is increased, as previously reported by $\mathrm{Bu}$ and Russo. ${ }^{20}$ However, up to the largest probes studied, there is no probe size studied here for which $\eta_{\mu}=\eta$ for the fast or intermediate mode at large polymer concentrations.

If one identified the lower-concentration intermediate mode (filled squares, $c<6 \mathrm{~g} / \mathrm{l}$ ) with the large-concentration slow mode (open squares, $c>6 \mathrm{~g} / \mathrm{l}$ ) rather than with the large concentration intermediate mode (filled squares, $c>6 \mathrm{~g} / \mathrm{l}$ ), then $\bar{\Gamma} \eta$ of the slowest observed mode of the $35 \mathrm{~nm}$ spheres would be independent of concentration over the full range studied. However, a Stokes-Einsteinian behavior that obtained for small but not large probes cannot be the largeprobe limiting behavior that is sometimes envisioned.

\section{DISCUSSION}

Time moments provide a physical parametrization of the mode structure of light scattering spectra. The time moment description differs from the $(\theta, \tau, \beta)$ description of Streletzky et al. ${ }^{13-18}$ in that time moments depend simply on $q^{2}$ and have the simple dimensions (time). ${ }^{1}$ The above data and reanalysis speak to several physical questions, and suggest a partial model for the observed phenomenology.

First, probe and polymer modes are not the same. Over limited concentration ranges particular polymer and probe modes may have the same relaxation time. However (Fig. 4), 


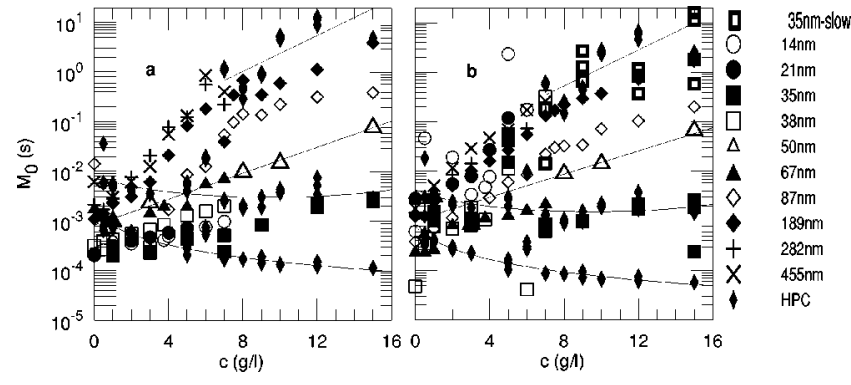

FIG. 9. $M_{0}$ for the (a) short-lived and (b) intermediate modes of light scattering spectra of polystyrene probes in HPC:water, and the three modes found in spectra of HPC:water, as functions of polymer concentration.

$M_{0}$ for probes generally increases with increasing polymer $c$, while $M_{0}$ of the two shorter-lived polymer modes falls or remains constant if $c$ is increased. The concentration dependences of the probe and polymer modes thus are not the same. The fast and intermediate probe modes therefore do not reflect probes moving in unison with polymer matrix, even though the time scales of the modes may roughly coincide.

This result is demonstrated by Fig. 9, which shows $M_{0}$ of the probes and the polymer matrix. Figure 9(a) shows the shorter-lived probe modes, while Fig. 9(b) shows the longerlived probe modes. Above ca. $4 \mathrm{~g} / 1 \mathrm{HPC}, M_{0}$ for the fast and intermediate modes are very nearly equal, so the right-hand sides of the two figures are similar.

In Fig. 9(a), lines to guide the eye mark polynomial fits to $M_{0}$ of the three polymer modes, and a simple exponential fit to $M_{0}$ of the $50 \mathrm{~nm}$ probes' shorter-lived mode. For small $(R \leqslant 50 \mathrm{~nm})$ probes, $M_{0}$ increases modestly with increasing $c ; d \log \left(M_{0}\right) / d c$ is approximately $0.13 \mathrm{l} / \mathrm{g}$. For larger probes, $M_{0}$ has three distinct regimes, namely a dilute regime in which $M_{0}$ increases slowly with increasing $c$, a nondilute regime in which $M_{0}$ increases more rapidly with increasing $c$, and a concentrated regime in which the increase in $M_{0}$ is again slow. The transition from the dilute to the nondilute regime is found when the probe $M_{0}$ becomes significantly larger than $M_{0}$ of the polymer intermediate mode. The transition from the nondilute to the concentrated regime is found at 6-7 g/l HPC, which is the concentration range in which the polymer slow mode becomes apparent. Only the 87 and $189 \mathrm{~nm}$ probes were taken to concentrations large enough to be in the concentrated regime. For the 455 and $282 \mathrm{~nm}$ probes, the dilute regime includes at most a few lowestconcentration points.

Figure 9(b) shows $M_{0}$ of the probe intermediate relaxation. For small $(R<50 \mathrm{~nm})$ probes, $M_{0}$ is somewhat scattered. For large probes, $M_{0}$ increases with increasing $c$. The intermediate and slow modes of the $35 \mathrm{~nm}$ probes have $M_{0}$ close, respectively, to $M_{0}$ of the intermediate and slow modes of the HPC itself.

Second, the apparent transition ${ }^{6}$ in the concentration dependence of $\eta$ of HPC:water solutions is echoed by transitions in probe behavior. The original question was whether the rheological transition at $c_{t}$ is real or a numerical fitting artifact. We find changes near $c_{t}$ in the behavior of $M_{0}$. The appearance of a transition near $c_{t}$ in independent physical properties supports the interpretation that the rheological transition is real.

Streletzky and Phillies ${ }^{16,18,19}$ have previously reported probe diffusion data showing transitions near $c_{t}$. They worked with large $(50,87$, and $189 \mathrm{~nm})$ probes, and characterized their spectra using Eq. (3) and parameters $\theta$ and $\beta$. For the broad mode, $\theta_{b}$ is independent of $c$ for $c<c_{t}$, but falls markedly with increasing $c$ for $c>c_{t}$. For the same mode, $\beta_{b}$ falls with increasing $c$ for $c<c_{t}$, but is nearly constant or increases slowly with increasing $c$ for $c>c_{t}$. The slope $d\left(\theta_{b} \eta\right) / d c$ also declines markedly near $c_{t}$. For $c<c_{t}$ the broad mode amplitude increases markedly with increasing $c$, while above $c_{t}$ this amplitude is nearly constant. These changes in concentration dependence all happen very nearly at $c_{t}$. Streletzky and Phillies ${ }^{16,18,19}$ therefore proposed that their probe diffusion results give independent experimental evidence that there is a rheological transition at $c_{t}$.

We extended earlier work by measuring $M_{0}$ for small 35 $\mathrm{nm}$ probes and by computing $M_{0}$ for other probes from previously determined $\theta, \beta$ parameters. With the 87 and $189 \mathrm{~nm}$ probes, there is (Fig. 9) near $c_{t}$ a diminution in the slope of $\log \left(M_{0}\right)$ against $c . \bar{\Gamma} \eta$ for the large-probe intermediate mode (Fig. 8) increases with increasing $c$, but more rapidly for $c$ $<c_{t}$ than for $c>c_{t}$. The $35 \mathrm{~nm}$ probes gain a new, very slow, relaxational mode at concentrations above a narrow concentration range near $c_{t} . \bar{\Gamma} \eta$ of the $35 \mathrm{~nm}$ probe intermediate mode increases drastically over a narrow range of concentrations near $c_{t}$, but increases much more gradually at all other concentrations. For all probe sizes, between $4 \mathrm{~g} / \mathrm{l}$ and $c_{t}$ the $M_{0}$ of the sharp and broad modes become approximately equal. Below $4 \mathrm{~g} / \mathrm{l} \mathrm{HPC}$, the $M_{0}$ of the two modes differ from each other.

The above all mark qualitative changes in probe dynamics occurring at $c \approx c_{t}$. We find here the new result that the rheological transition at $c_{t}$ appears in spectra of small as well as large probes. Furthermore, the apparent transition survives reparameterization of the spectral lineshape from $(\theta, \beta)$ to a more simply dimensioned $M_{0}$. We conclude that the viscosity transition at $c_{t}$ is a real physical phenomenon, in agreement with Streletzky et al. ${ }^{13-19}$ and offer new experimental data and a novel method for characterizing spectra in support of our conclusion.

Third, our results identify a single important distance scale in HPC:water solutions, namely a concentrationindependent length in the range 50-70 $\mathrm{nm}$. The distance scale manifests itself in a series of disparate observations. We find no indication that the distance scale depends substantially on polymer concentration, though a weak concentration dependence cannot be ruled out.

In particular, for small probes below $c_{t}$ the broad mode is longer lived (larger $M_{0}$ ) than the sharp mode, while for large probes the broad mode is shorter lived than the sharp mode. The diameter separating small from large probes is about $50 \mathrm{~nm}$. For small probes the relative amplitudes of the sharp and broad modes depend strongly on the scattering vector. At low $q$, the broad mode dominates. At larger $q$ the sharp mode dominates. The crossover is found at a wave vector $q_{c}$ corresponding to distances $q_{c}^{-1} \approx 70 \mathrm{~nm}$. The mean relaxation rate of the small-probe broad mode suddenly in- 
creases markedly at a wave vector corresponding to distances $q^{-1} \approx 50 \mathrm{~nm}$.

Finally, the probe intermediate mode becomes much more strongly concentration dependent when it becomes longer lived than the polymer intermediate mode. This circumstance arises at different concentrations, depending on the probe size. For a probe of the correctly selected size, the probe and polymer intermediate modes are equally long lived at the rheologically interesting concentration $6 \mathrm{~g} / \mathrm{l}$ $\approx c_{t}$. The size of a correctly selected probe is approximately $50 \mathrm{~nm}$.

Thus, the sharp and broad mode relaxation time crossover, the $q$ dependence of the sharp and broad mode amplitudes and of the small-probe broad-mode relaxation rate, and the crossover of the probe and polymer intermediate modes all reference a concentration-independent length scale near $50 \mathrm{~nm}$. As a characteristic length, $50 \mathrm{~nm}$ has a physical significance: It is very nearly equal to the $49 \mathrm{~nm}$ mean hydrodynamic radius found by Yang and Jamieson ${ }^{5}$ for a hydroxypropylcellulose of this molecular weight.

Our conclusions are consistent with the conclusions of Streletzky et al. ${ }^{13-18}$ These workers found distinctive smallprobe and large-probe behaviors in the parameters $\theta, \beta, \tau$, the crossover between small and large again being found for probes near $50 \mathrm{~nm}$ in size. Over a 15 -fold change in polymer concentration, they found no change in the apparent length scale.

Fourth, characterization of complex modes with their mean relaxation time $M_{0}$ rather than with $\theta$ and $\beta$ eliminates prior complexities. Ngai and Phillies ${ }^{23}$ and Streletzky and Phillies ${ }^{17}$ compared $\theta$ and $\beta$ from the concentration dependences of probe diffusion and the viscosity with predictions of the Ngai ${ }^{24}$ coupling-scaling model. This model had previously been applied to a variety of other complex systems. ${ }^{24,25}$ The Ngai ${ }^{24}$ model provides a general description for relaxations of anharmonically coupled dynamic units. At very short times, the units are proposed to relax independently, leading to pure-exponential relaxations. At longer times, couplings between the units have had time to become effective, and the model proposes that relaxations become stretched exponentials in time. The Ngai model predicts a relationship between the crossover time separating short- and long-time behavior, the characteristic times of the exponential and stretched-exponential relaxations, and the coupling strength as revealed by the shape parameter $\beta$.

Earlier papers ${ }^{17,23}$ found that the coupling model approximately describes the concentration dependences of the broad mode of large probes and the sharp mode of small probes, but not the sharp mode of large probes or the broad mode of small probes. A prediction that works for one mode of some probes and the other mode of other probes seems complex and unconvincing. However, if the probe spectral modes are identified by their mean relaxation times rather than their initial relaxation rates, as we did here, a much simpler description arises. For all probes, the Ngai couplingscaling model describes parameters for the probe fast mode, but not for the probe intermediate mode. The mean relaxation time gives a simpler description than does the initial decay rate.
Finally, our results suggest a plausible model for the observed dynamics. For $c<c_{t}$, we propose that polymer dynamics are dominated by hydrodynamic interactions between the chains. At $c_{t}$, we propose that our solutions have a dynamic transition. Specifically, we propose that our data are consistent with the appearance of a generalized Kivelson glass (Ref. 28) in our HPC solutions at concentrations $c>c_{t}$. The generalized glass differs from the glass envisioned by Kivelson et al., in that (i) our system is a solution, not a neat liquid, so concentration is a controlling variable, and (ii) our glass-forming molecules are random-coil polymers, not spheroids, so the factors inhibiting unlimited cluster growth are not the icosahedral packing constraints discussed by Kivelson et al.

For lower concentrations the hydrodynamic model of nodilute polymer solutions has been analyzed previously. ${ }^{26,27}$ It predicts a stretched-exponential concentration dependence for $\eta$. This concentration dependence is indeed observed ${ }^{6}$ for $\eta$ for $c<c_{t}$. The model has a characteristic length scale, given by a hydrodynamic size of a polymer coil. For HPC of approximately our molecular weight, Yang and Jamieson ${ }^{5}$ report $1 /\left\langle R_{h}^{-1}\right\rangle \approx 49 \mathrm{~nm}$, which is the $50 \mathrm{~nm}$ characteristic length found above in this system.

The original Kivelson ${ }^{28}$ glass model treated a onecomponent fluid. In the Kivelson model, in addition to crystalline solid and liquid phases, the molecules of a Kivelson glass form equilibrium clusters that are not crystallites of the solid phase. Instead, the equilibrium clusters have the signal feature that their structure is not space filling. Clusters are energetically constrained from growing beyond a characteristic size, so they cannot grow into a crystalline phase. If the liquid is supercooled, more clusters form, leading at low temperature to a glass: a highly viscous packing of perhaps jammed clusters, perhaps with more fluid molecules filling interstices between clusters. The clusters cannot grow to fill space, so at high temperatures they can be more stable than the liquid without creating a thermodynamic paradox. Clusters thus can exist, not only in the supercooled liquid, but also in the normal liquid above the melting point. The model thereby explains high-temperature features of glass-forming liquids.

We generalize the Kivelson model to solutions, envisioning glass formation via growth-constrained clusters of polymer molecules. In a solution, cluster formation is driven by concentration rather than temperature. Just as onecomponent clusters can be stable at temperatures above the melting point of the crystal, so solution clusters can be stable at solute concentrations below the solubility limit of the solid but above some limiting concentration $c_{c}$ for cluster formation. $c_{c}$ is directly analogous to the "critical micellar concentration" for micelle formation. Above $c_{c}$, the presence of clusters affects the solution dynamics.

The critical concentration for cluster formation is here identified with the transition concentration $c_{t}$. Our data are consistent with cluster formation. Namely, (i) above $c_{t}$, probe-free HPC solutions gain a third, slowly relaxing mode corresponding to the diffusion of clusters. The appearance of the slow polymer mode is discontinuous. (ii) Probes that are smaller than clusters could be entrained within a cluster. En- 
trained probes would diffuse at the same rate as the clusters carrying them. At $c_{t}$, the $35 \mathrm{~nm}$ spheres-the only small probes studied at $c>c_{t}$ - gain a slow mode in their light scattering spectra. The relaxation times of the polymer and small-probe slow modes are the same, as expected for probes entrained in clusters; see Figs. 2 and 9. (iii) By analogy with micelle formation, the concentration of clusters would grow smoothly from zero as $c$ increases above $c_{c}$, causing transport coefficients to deviate continuously with increasing $c>c_{t}$ from the behavior expected in the absence of cluster formation. In contrast, at a phase transition, dynamic properties show discontinuous changes. We find that $\eta$ and $M_{0}$ show the changes expected for cluster formation at $c_{t}$; above $c_{t}$ their concentration dependences show changes in slope but not discontinuous changes in value.

The measured temperature dependences of the fast and intermediate probe modes provide substantial additional support for the model proposed here. At all polymer concentrations, the mean relaxation rates $M_{0}^{-1}$ depend on temperature via $T / \eta_{0}, \eta_{0}$ being the viscosity of the solvent. $\eta_{0}$ is the transport parameter reflecting solvent motion. For $c<c_{t}$, its presence in the temperature dependence is explicitly predicted by the hydrodynamic ${ }^{26,27}$ model. For $c>c_{t}$, an extended hydrodynamic model that included hydrodynamic interactions with diffusing clusters would continue to share the temperature dependence of $\eta_{0}$. On the other hand, if the cluster lifetime contributed to the relaxation rates, for example because probes were able to advance when clusters fell apart and left solution-filled void spaces, the temperature dependence for probe motion would be determined in part by the activation energy for cluster formation and decomposition, which except by coincidence would differ from the activation energy for the solvent viscosity. The absence of a temperature dependence other than that of the solvent viscosity indicates that the processes controlling probe motion are all dominated by the solvent viscosity, i.e., they are all processes dominated by solvent-mediated hydrodynamic interactions.

Are experimental data quantitatively consistent with cluster formation? Do probes diffuse at the rates expected for a cluster-forming polymer solution? To answer this question we would need a quantitative model for diffusion and momentum transport in a mixture of overlapping polymer coils and long-lived chain clusters. Such a model does not appear to be available.

Our measurements give only limited physical information on the physical nature of the clusters. The data here do not prove that clusters are equilibrium objects localized in position space. The spectral features being interpreted here as clusters could alternatively arise from persistent dynamic structures, having a characteristic length scale ca. $50 \mathrm{~nm}$, whose transient permanence arises from the solution dynamics rather than from thermodynamic variables. Indeed, the lack of a significant increase in the normalized static light scattering intensity $I / c$, as the transition concentration is crossed, strongly indicates that the slow mode does not come from localized regions of very high concentration. HPC does have a liquid-liquid crystal phase transition in a very different part of its phase diagram, suggesting that its chain seg- ments are predisposed to align, but we have no information on chain alignments here.

As an alternative to finding the mean lifetime of the relaxation modes, one could envision an analysis that extracted a terminal diffusion coefficient $D_{t}$ from the terminal behavior of $g^{(1)}(t)$. Such an analysis, which is equivalent to measuring the mean-square distance $\left\langle x^{2}\right\rangle$ traveled by diffusing particles over a long time $t$ and taking

$$
D_{t}=\frac{\left\langle x^{2}\right\rangle}{t},
$$

implicitly presumes that the spectra have a terminal pureexponential mode. This presumption is not consistent with our experimental data on small probes at low concentrations and on all probes at elevated concentrations. We find that no mode is longer lived than a mode that decays as a stretched exponential in time.

A standard argument indicates that if there is a welldefined longest length scale $\xi$ in a system, then diffusion over distances much longer than $\xi$ will be a random walk, whose diffusive motions satisfy Eq. (12) and whose $g^{(1)}(t)$ is a simple exponential. Conversely, because the terminal relaxational mode that we observe experimentally does not decay as a pure exponential, we conclude that the systems we study do not have a single longest length scale $\xi$. We do observe a characteristic length $\approx 50 \mathrm{~nm}$, which is the size of a polymer coil. Because hydrodynamic interactions are long range (the Oseen tensor is an $r^{-1}$ interaction), the observed characteristic length does not necessarily supply the longest length scale. Instead, our results suggest that in these systems there are interesting dynamic behaviors on all length scales, which-once one has reached scales much longer than the characteristic length-would appear obliged to extend to larger distance scales in a self-similar manner.

It is legitimate to inquire if the transition seen here is an "entanglement" transition in the sense of, e.g., Graessley. ${ }^{29}$ We actually do not measure individual chain motions directly and hence cannot say whether, e.g., the slow mode corresponds to topological restrictions on chain motion. However, the entanglement transition as envisioned in Ref. 29 is viewed to be a phenomenon arising from universal features of polymer chains. In contrast, the viscometric transition ${ }^{6}$ that originally drew our attention to this system is nonuniversal, being found various systems at very different values of $c[\eta]$ and being absent in other concentrated polymer systems. ${ }^{30}$ The viscometric transition discussed here thus does not appear to have the properties expected of an entanglement transition.

\section{ACKNOWLEDGMENT}

The partial support of this work by the National Science Foundation under Grant No. DMR99-85782 is gratefully acknowledged.

${ }^{1}$ H. A. Swensen, C. A. Schmitt, and N. Thomson, J. Polym. Sci., Part C: Polym. Symp. 11, 243 (1965).

${ }^{2}$ S. Aharoni, Macromolecules 16, 1722 (1983).

${ }^{3}$ M. Mustafa and P. S. Russo, J. Colloid Interface Sci. 129, 240 (1989). 
${ }^{4}$ M. B. Mustafa, D. L. Tipton, M. D. Barkley, P. S. Russo, and F. D. Blum, Macromolecules 26, 370 (1993).

${ }^{5}$ T. Yang and A. M. Jamieson, J. Colloid Interface Sci. 126, 220 (1988).

${ }^{6}$ G. D. J. Phillies and C. A. Quinlan, Macromolecules 28, 160 (1995).

${ }^{7}$ F. R. Hallett and A. L. Gray, Biochim. Biophys. Acta 343, 648 (1974).

${ }^{8}$ D. N. Turner and F. R. Hallett, Biochim. Biophys. Acta 451, 305 (1976).

${ }^{9}$ T. C. Laurent, I. Bjork, A. Pietruskiewicz, and H. Persson, Biochim. Biophys. Acta 78, 351 (1963).

${ }^{10}$ T. C. Laurent and H. Persson, Biochim. Biophys. Acta 83, 141 (1964).

${ }^{11}$ W. Brown and R. Rymden, Macromolecules 19, 2942 (1986).

${ }^{12}$ P. S. Russo, M. Mustafa, T. Cao, and L. K. Stephens, J. Colloid Interface Sci. 122, 120 (1987).

${ }^{13}$ K. A. Streletzky and G. D. J. Phillies, J. Chem. Phys. 108, 2975 (1998).

${ }^{14}$ K. A. Streletzky and G. D. J. Phillies, J. Polym. Sci., Part B: Polym. Phys. 36, 3087 (1998).

${ }^{15}$ K. A. Streletzky, Ph.D. thesis, Worcester Polytechnic Institute, 1998.

${ }^{16}$ K. A. Streletzky and G. D. J. Phillies, Macromolecules 32, 145 (1999).

${ }^{17}$ K. A. Streletzky and G. D. J. Phillies, J. Phys. Chem. B 103, 1811 (1999).

${ }^{18}$ K. A. Streletzky and G. D. J. Phillies, "Optical probe study of solutionlike and melt-like solutions of high molecular weight hydroxypropylcel- lulose," in Scattering from Polymers, edited by B. S. Hsiao [American Chemical Society Symposium Series 739, 297-316 (2000)].

${ }^{19}$ G. D. J. Phillies and K. A. Streletzky, Recent Res. Dev. Phys. Chem., Transworld Research Network 5, 269 (2001).

${ }^{20} \mathrm{Z}$. Bu and P. Russo, Macromolecules 27, 1187 (1994).

${ }^{21}$ G. D. J. Phillies and R. O’Connell (unpublished).

${ }^{22}$ G. D. J. Phillies, J. Phys. Chem. 99, 4265 (1995).

${ }^{23}$ K. L. Ngai and G. D. J. Phillies, J. Chem. Phys. 105, 8385 (1996).

${ }^{24}$ K. L. Ngai, in Disorder Effects in Relaxation Processes, edited by R. Richert and A. Blumen (Springer, Berlin, 1994), pp. 89-150.

${ }^{25}$ Proceedings of the International Discussion Meeting on Relaxations in Complex Systems, edited by K. L. Ngai and G. B. Wright [J. Non-Cryst. Solids 131-133 (1991)].

${ }^{26}$ G. D. J. Phillies, J. Chem. Phys. 116, 5857 (2002).

${ }^{27}$ G. D. J. Phillies, Macromolecules 31, 2317 (1998)

${ }^{28}$ S. A. Kivelson, X. Zhao, D. Kivelson, T. M. Fischer, and C. M. Knobler, J. Chem. Phys. 101, 2391 (1994).

${ }^{29}$ W. W. Graessley, Adv. Polym. Sci. 16, 1 (1974).

${ }^{30}$ G. D. J. Phillies, Macromolecules 28, 8198 (1995). 